\title{
MICROARRAY ELECTRODES FOR IMPEDANCE IMAGING AND ELECTRICAL CHARACTERIZATION OF EX-VIVO HUMAN LIVER METASTASES FROM COLORECTAL CANCER
}

\author{
Michael Karnes ${ }^{1}$, Ryan Snodgrass ${ }^{1}$, Emily Sequin ${ }^{1}$, Charles Hitchcock ${ }^{2}$, Edward Martin, $\mathrm{Jr}^{3}$, \\ Shawnn D. Nichols ${ }^{3}$, Carl Schmidt ${ }^{3}$, Sherif Abdel-Misih ${ }^{3}$, Mark Bloomston ${ }^{3}$, Vish Subramaniam ${ }^{1 *}$, \\ Shaurya Prakash ${ }^{1 *}$ \\ ${ }^{1}$ Department of Mechanical and Aerospace Engineering, The Ohio State University, Columbus, Ohio, USA \\ ${ }^{2}$ Department of Pathology, The Ohio State University, Columbus, Ohio, USA \\ ${ }^{3}$ Department of Surgical Oncology, The Ohio State University, Columbus, Ohio, USA
}

\begin{abstract}
An image of morphologically distinct regions in resected human liver tissue containing metastatic colorectal cancer was generated using micro-electrical impedance spectroscopy ( $\mu$-EIS), providing quantitative information on tissue properties and structure, reflecting local gradients in electrical conductivity and permittivity. These impedance measurements were able to differentiate between regions of tumor and non-tumor tissue, allowing for the direct inferences of tissue microstructure and disease state. This work consequently provides major advancements toward a microscale instrument for the real time quantitative differentiation of tumor and non-tumor tissues in the surgical field, aiding the surgeon in selecting proper margins, which have proven to be critical in the long term survival of cancer patients.
\end{abstract}

\section{INTRODUCTION}

The mechanisms by which normal cells in the colon mutate to become cancerous are still being elucidated. As the solid tumor grows, cells will migrate from the primary cancer site to distant organs in the body as metastatic disease. In colorectal cancer, the liver is a common site for metastatic spread and surgical resection is the gold standard in managing such patients. A successful surgery is defined as the complete resection of the tumor with a finite rim of healthy or normal surrounding tissue, referred to as the surgical margin. It is within this rim that the transition from tumor to normal tissue occurs, and is termed the tumor interface. In colorectal liver metastases, surgeries with margins of $<1 \mathrm{~mm}$ pose a significant risk to patients, with a 5-year post-treatment survival rate of only $17 \%$, compared to $68 \%$ for surgeries with a margin of $>1 \mathrm{~mm}$ [1]. Current surgical techniques rely on the surgeon's visual and tactile evaluation of the liver, augmented with ultrasound assessment, to identify the target tumors, estimate the margin, and plan the resection. Margins are then confirmed via optical microscopy and pathology post-surgery.

It has been demonstrated that tumors have distinct electrical impedances as compared to surrounding normal tissue [2]. For biological samples, the electrical impedance is often referred to as bioimpedance, and describes the complex electrical resistance of a tissue to a voltage or current signal, comprised of charge flow and charge storage within the sample. Commonly these properties are reported as an admittance, $Y=K \sigma+K \varepsilon_{0} \varepsilon_{r}$; where, $\sigma$ is the electrical conductivity, and $\varepsilon_{r}$ is the relative permittivity of the sample. $K$ is a lumped geometric probe parameter, describing the current path in the sample and probe-sample interactions. The lumped geometric probe parameter is found by calibrating the probe in a range of solutions with known conductivities likely matching the tissue properties [3].

In general, the response of tissues to AC electric fields is determined by tissue architecture and composition. However, certain tissue characteristics become dominant for charge transport, i.e. current flow in specific frequency ranges. It is generally believed that in the $\alpha$ dispersion range, $1 \mathrm{~Hz}-100 \mathrm{kHz}$, counterion polarization along the cell membrane dominates the measured impedance. From $100 \mathrm{kHz}-1 \mathrm{MHz}$, the $\beta$ dispersion range, the extra cellular fluid is believed to be the major contribution to the measured impedance $[4,5]$.

Previously, electrical or electrochemical impedance spectroscopy (EIS) measurements have been successfully used for imaging breast and prostate cancers, distinguishing tumor from normal tissue [5]. The purpose of this paper is to demonstrate the use of two $\mu$-EIS measurement probes, one in a single 4-wire configuration used in a physical translation set-up and the second in a microarray configuration to quantify the differences between tissue types. Based on the quantification, an image contour was generated that provides a quick visual guide to tissue morphology and structure as mediated by gradients in electrical properties.

\section{METHODOLOGY}

Measurements were conducted on excised human liver tissue containing colorectal metastases as part of a study (protocol \#2013C0009) approved by the Institutional Review Board (IRB) at The Ohio State University. After surgical resection, the tissue was immediately transported in a sealed container to the Department of Pathology for sectioning relevant tissue for clinical requirements. Residual remnant tissue was obtained for inclusion into this study, and EIS measurements were collected with all measurements being initiated within 2 hours of tissue removal from the patient.

The physical dimensions and initial weight of the tissue were measured along with ambient temperature. Digital photographs were taken and the tissue marked with India ink as a reference for registration of subsequent measurement locations. The tissue was then placed on a computer controlled stage, where the single 4wire $\mu$-EIS probe was moved to the origin mark and the coordinates zeroed. From here, the probe was moved to the desired measurement locations, and measurement coordinates were recorded. The probe, as shown in Figure 1, penetrated the tissue to a depth of $4 \mathrm{~mm}$.

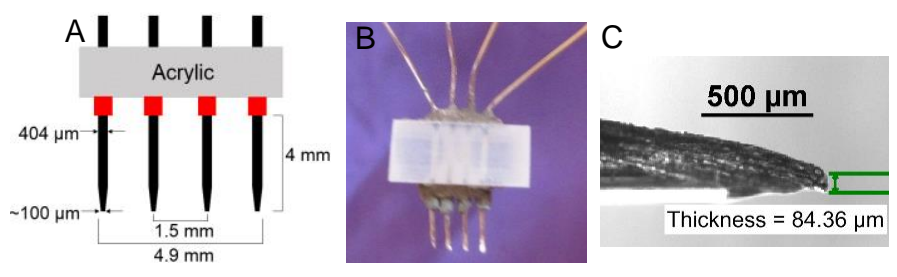

Figure 1: A. Probe schematic with dimensions. B. Digital image showing unit-cell for a 4-wire probe used for $\mu$-EIS. C. Optical microscope image at $4 x$ magnification to show the mechanically sharpened tip which allows easy penetration of tissue.

Solid-State Sensors, Actuators and Microsystems Workshop Hilton Head Island, South Carolina, June 8-12, 2014 
The 4-wire probe was made using 99.9\% pure (Alfa Aesar, MA, USA) Platinum (Pt) wires. In this setup, a $30 \mu \mathrm{A}$ RMS current was injected by the outer two electrodes, while voltage is measured by the inner two. The entire probe is $\sim 4.9 \mathrm{~mm}$ in length, and has a pin pitch of $1.5 \mathrm{~mm}$. Once all measurements had been completed, the tissue weight was recorded again to determine the weight lost, most likely due to tissue desiccation.

The recorded impedance data was post-processed using MATLAB. As a representative analysis example, using the marked origin, and location coordinates, the probe footprints were plotted onto the photograph, shown in Figure 2. Each point was then classified as normal, tumor, or interface by measuring the number of pixels between the center of the measurement location to the closest tumor edge, as shown in Figure 2. Points within a half probe length radius $(\sim 2.45 \mathrm{~mm})$ of a tumor edge were labeled interface. Points within the tumor and greater than a half probe length away from the edge were labeled tumor; while those more than half probe length away from the visible tumor edge on the normal tissue side were classified as normal tissue. The classification of normal and tumor tissue for quantification of liver tissue has also been reported previously $[2,3]$.

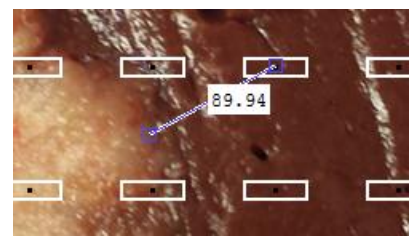

Figure 2: Photograph of specimen liver tissue with probe footprint plotted. The figure also shows the pixel distance measuring tool illustrating classification scheme for determining normal, tumor, or interface region in ex vivo human liver tissue.

The second probe, a microarray probe comprised of a two row arrangement of 16 electrodes each with a $1.3 \mathrm{~mm}$ pin pitch and $460 \mu \mathrm{m}$ electrode or pin diameter as shown in Figure 2. The microarray probe eliminates the need to translate the 4-wire probe and allows for multiple measurements within an area defined by the probe footprint. A microcontroller (Arduino Mega 2560) controls four 32 pin multiplexers enabling the switching of active electrodes (4 per measurement) for sequential measurement of 26 locations without probe translation within a total probe footprint of $\sim 2 \mathrm{~cm}$ length and $\sim 3 \mathrm{~mm}$ width. Measurements with the microarray probe were conducted on porcine muscle tissue phantoms as opposed to human liver tissue due to the need to develop proof-of-concept and reliable probe operation before IRBbased protocol implementation on human liver tissue.

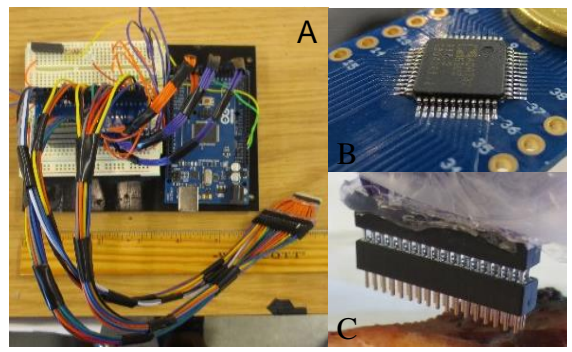

Figure 3: A. Complete electrode array, with probe, microcontroller, and multiplexer, in total taking up a $15 \mathrm{~cm}$ square (black supporting tray). B. A zoomed-in image of the 32 pin multiplexer. C. 32-electrode array with wires covered by plastic bag to prevent tissue contact and thereby contamination from likely tissue infections, for use with human tissue.
For all measured values, impedance data was recorded using a commercially available potentiostat (Interface 1000 from Gamry Instruments, PA, USA). A constant current injection of $30 \mu \mathrm{A}$ RMS amplitude over a frequency range of $100 \mathrm{~Hz}-1 \mathrm{MHz}$ was used. Frequencies below $100 \mathrm{~Hz}$ are known to induce electrode polarization, and therefore were not included $[3,6]$.

Random error from repeated measurements was estimated to be $\sim 4 \%$ of the measured value with instrument error being at most $\sim 1 \%$ of the measured value. The data is presented as an arithmetic mean for the specific tissue type (normal or tumor). The errors bars represent the standard deviation of the mean. The measured phase and the results of a single tail Student t-test for a $95 \%$ confidence interval are also presented.

\section{RESULTS AND DISCUSSION}

Figure 4 shows conductivity, $\sigma$ of the data collected compared to previously published values. Note that the mean normal tissue electrical conductivity is lower by nearly an order of magnitude compared to that of the tumor; however, the variation in the tumor tissue is $60 \%$ greater than that of the normal as indicated by the error bars denoting the standard deviation. This is not surprising since it is known that tumors are not homogeneous, but consists of tissues at various disease states.

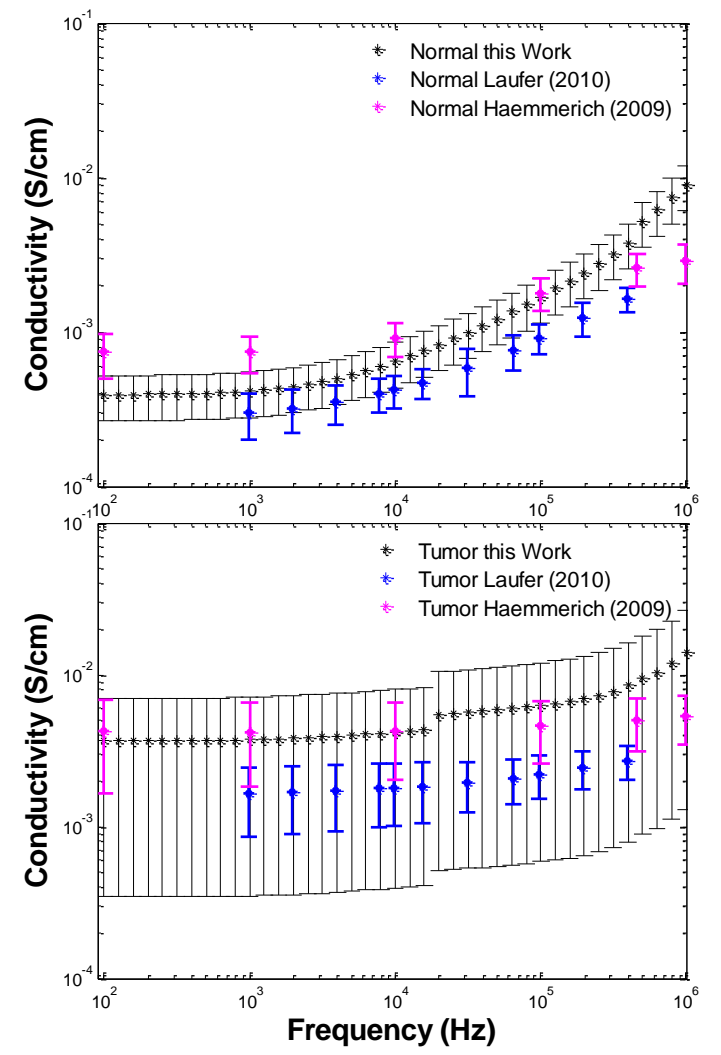

Figure 4: A. Normal tissue conductivity (mean $\pm S D$ ) vs. frequency for normal tissue determined by methods previously reported. The plot also shows conductivity values reported by Laufer (2010) and by Haemmerich (2009). B. Tumor tissue conductivity (mean $\pm S D$ ) vs. frequency for normal tissue determined by methods previously reported, and conductivity values reported by Laufer (2010) and by Haemmerich (2009). It should be noted that the two studies cited here are the only two peer-reviewed papers available on electrical properties of human liver tissue with metastatic disease from colorectal cancer. 
Figure 5 shows the phase plots for the measured impedance with data reported as the arithmetic mean and standard deviation of the data points. Both normal and tumor tissue have a negative phase angle across the entire measured frequency range, implying that the tissue shows behavior analogous to a capacitive response. The tumor tissue produced a smaller phase shift, having a maximum magnitude of $10^{\circ}$, with the normal tissue at a $37^{\circ}$ phase shift with statistical significance verified for a $95 \%$ confidence interval by the student t-test.

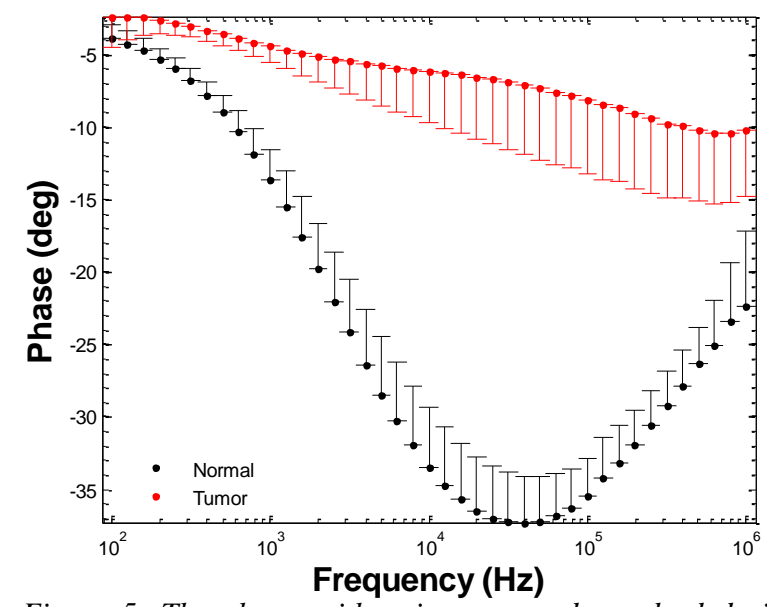

Figure 5: The phase arithmetic mean and standard deviation. Note that the tumor shows a smaller phase shift in the impedance data compared to normal tissue, with the negative phase angle implying a behavior analogous to a capacitive response.

Figure 6 demonstrates the ability of the $\mu$-EIS method to distinguish tissue structural features by generating an equivalent impedance image of the tissue. Figure 6A shows a digital photograph of the specimen liver with measurement locations. The contour plot, in Figure 6B shows the phase shift at each measurement location at $\sim 25 \mathrm{kHz}$, as a representative example. The contour image shows a discernible difference between the tumor and normal tissue with a gradient at the interface region. Furthermore, visual, qualitative comparison between Figures 6A and $6 \mathrm{~B}$ also shows locations where blood vessels or other structures may have been present in vivo but do not exist (appear as holes or dark spots in digital images) in the ex vivo specimen.

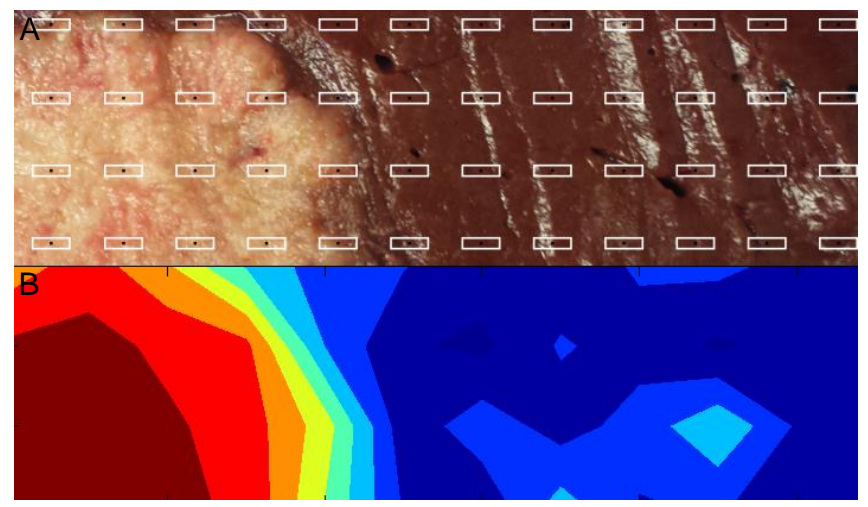

Figure 6: A. Digital image of liver sample. B. Contour plot of the specimen phase shift at $\sim 25 \mathrm{kHz}$, with a visual representation of tissue structure and disease state. The scale goes from red at the highest magnitude of phase to blue at the lowest.

For the multiplexed probe, a representative data set for the porcine muscle tissue phantom is shown in Figure 7. With this probe, each placement yields measurements at 26 unique points within the probe footprint. The fat (as annotated on the digital image) has distinctly higher impedance than the muscle tissue. The point of transition is not clear likely due to the probe being in both parts of tissue during the same measurement, and requires further investigation.

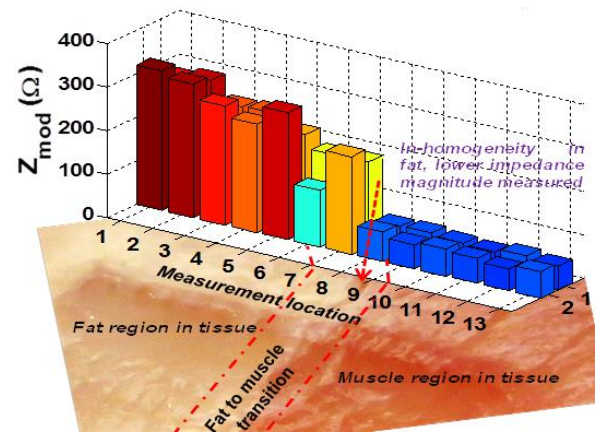

Figure 7: A bar graph plot showing magnitude of impedance and comparing the measurement locations to a visual representation of the tissue phantom.

\section{CONCLUSION}

Two $\mu$-EIS probes were demonstrated for use in quantifying tissue types based on electrical impedance data. The phase shift in the impedance data was organized into an image contour to enable impedance imaging of the tissue to depict tumor from normal tissue. With the ability to reach finer spatial resolution underway in other experiments, the method shows promise for accurate tissue microstructure delineation.

\section{ACKNOWLEDGEMENTS}

The Authors acknowledge support from Karen Bellman and Benjamin Shurtz in data collection. Partial financial support from Samsung, Inc. is also recognized.

\section{REFERENCES}

[1] T.M. Pawlik, C.R. Scoggins, D. Zorzi, E.K. Abdalla, A. Andres, C. Eng, S.A. Curley, E.M. Loyer, A. Muratore, G. Mentha, L. Capussotti, and J.N. Vauthey, "Effect of surgical margin status on survival and site of recurrence after hepatic resection for colorectal metastases", Annals of Surgery, 241, 5 (2005).

[2] D. Haemmerich, D.J. Schutt, A.S. Wright, J.G. Webster, and D.M. Mahvi, "Electrical conductivity measurement of excised human metastatic liver tumours before and after thermal ablation", Physiol. Meas., 24, 251, (2009).

[3] S. Laufer, A. Ivorra, V.E. Reuter, B. Rubinsky, S.B. Solomon, "Electrical impedance characterization of normal and cancerous human hepatic tissue", Physiol. Meas., 31, 995 (2010).

[4] Miklavčič, D., N. Pavšelj and F. X. Hart (2006). Electric Properties of Tissues. Wiley Encyclopedia of Biomedical Engineering, John Wiley \& Sons, Inc.

[5] K.R. Foster, H.P. Schwan, "Dielectric properties of tissues and biological material: a critical review", Crit. Rev. Biomed. Eng., 17, 25 (1989).

[6] Schwan, H. P. (1968), "Electrode Polarization Impedance and Measurements in Biological Materials", Annals of the New York Academy of Sciences, 148: 191-209.

\section{CONTACT}

*S. Prakash, tel: +1-614-688-4045; prakash.31@osu.edu

*V. Subramaniam, tel: +1-614-292-6096; $\underline{\text { subramaniam.1 @ osu.edu }}$ 